\title{
Dynamic Grouped Chip-Level Iterated Multiuser Detection Based on Gaussian Forcing Technique
}

\author{
F. Wathan, Student Member, IEEE, R. Hoshyar, Member, IEEE, and R. Tafazolli
}

\begin{abstract}
The brute-force MAP-based Chip-Level Iterated (CLi) Multiuser Detection techniques exploiting the newly proposed Low-Density Signature (LDS) structure have been shown to approach the performance of Single-User even at overloading condition of up to $200 \%$. However, their complexity is exponential to the number of interfering users in a chip that can be impractical. In this letter, by using Gaussian-Forcing (GF) technique, we trade-off performance and complexity and it results in a dynamic Grouped GF (G-GF) CLi MUD technique. We show via computer simulation, at the system with $200 \%$ condition, the loss of approximately $0.3 \mathrm{~dB}$ compared to its brute-force counterpart is observed while reducing the complexity to more than half.
\end{abstract}

Index Terms - Low-density signature, synchronous overloaded CDMA, chip-level iterated MUD, dynamic user grouping, Gaussian-forcing technique.

\section{INTRODUCTION}

$\mathbf{E}$ VERGROWING demand in contemporary communication is to increase the capacity. In this letter, the capacity is understood as the user capacity, defined as the maximum number of admissible users at any time-instants [1]. For interference-limited systems, e.g. Code-Division Multiple Access (CDMA) systems, the soft limit of the user capacity, in memoryless channel, is the processing gain, defined as $N$. Let $K$ and $\beta=K / N$ be the number of users in the system and the system loading, respectively. The system is called, underloaded, fully-loaded, and overloaded (or oversaturated), when the $\beta$ is lower than, equal to, and bigger than 1 , correspondingly.

The interference in CDMA systems is caused by the nonorthogonality of the (received) signatures, which can be overcome by using extensive signal processing on the signal space, for instance by using Multiuser Detection [2]. Nevertheless, overloaded condition possess a different problem as it makes at least two signatures to be linearly-dependent. This is the exact problem where most MUD technique simply fails to cope with even to meet a basic reliable communication, i.e. the Bit-Error Rate vanishes as the noise approaches zero.

Combined with optimum Spreading Signature for overloaded condition, namely Welch-Bound-Equality (WBE) based signatures [3], Optimum MUD, for example Minimum A Posteriori Probability (MAP) detector, can be used but its complexity prevent its practical implementation. Linear MUD, for instance Minimum Mean-Squared Error (MMSE) detector,

Manuscript received November 17, 2007. The associate editor coordinating the review of this letter and approving it for publication was G. Mazzini.

The authors are with the Mobile Communication Research Group, Centre for Communication Systems Research, University of Surrey, United Kingdom (e-mail: \{f.wathan, r.hoshyar, r.tafazolli\}@ surrey.ac.uk).

Digital Object Identifier 10.1109/LCOMM.2008.071931. can only mitigates the interference maximum of $N$ users and leave the $K-N$ excess users saturates [4].

Recently, Low-Density Signature Structure complemented with more-affordable MAP-based Chip-Level iterated (CLi) MUD has been shown to achieve performance very close to single-user even at $200 \%$ overloaded condition [5], [6]. In this letter, we concentrate a new technique that reduces the complexity of the MAP-based CLi MUD without losing too much of performance.

\section{SySTEM MOdeL}

Consider an uncoded $(N, K)$ symbol-synchronous CDMA system for uplink transmission over AWGN channel. Let $x_{k}$ and $\mathbb{X}_{k}$ be, respectively, the transmitted symbol with period of $T_{s}$ and the constellation alphabet for user $k$. Without loss of generality and for notation brevity, all users are assumed to take their symbol from the same normalized constellation alphabet, i.e. $\mathbb{X}_{k}=\mathbb{X}, \forall k=1, \ldots, K$ with unit average energy. The received signal sampled at $n$-th chip is given by

$$
y_{n}=\sum_{k=1}^{K} h_{k, n} x_{k}+\nu_{n}
$$

where $y_{n}$ and $\nu_{n} \sim \mathcal{C N}(0,1)$ are the received signal and the circularly symmetric gaussian noise, respectively, sampled at $n$-th chip. The effective signature is denoted as $h_{k, n}=$ $e^{j \theta_{k}} \sqrt{\mathrm{snr}_{k}} s_{k, n}$ for $k$-th user sampled at and comprises the complex gain experience by the symbol due to spreading $\left\{s_{k, n}\right\}_{n=1}^{N}$, user's power $\operatorname{snr}_{k}$ and the user-unique phase rotation $\theta_{k}$.

The LDS structure works by switching-off a large number of spreading chips and with intelligent arrangement, the number of interfering users per chip is also reduced. Let $\xi_{n}$ and $\zeta_{k}$ be the set of users participating in chip $n$ and the set of chips over which user $k$ spreads their symbol, respectively. Then, with LDS structure, the received signal of (1) is

$$
y_{n}=\sum_{k \in \xi_{n}} h_{k, n} x_{k}+\nu_{n}
$$

\section{Chip-Level Iterated MUD}

\section{A. Canonical Form}

Denote $\mathcal{G}(\mathcal{U}, \mathcal{C})$ as the underlying factor graph of un-coded $(N, K)$ CDMA systems, where the users $(\mathrm{k}=1, \ldots, \mathrm{K})$ and the chips $(\mathrm{n}=1, \ldots, \mathrm{N})$ are represented by variable nodes $u \in \mathcal{U}$ and function nodes $c \in \mathcal{C}$, respectively. An edge $e(k, n)$ exists if the received chip $y_{n}$ contains the symbol from user $k$ and is shown as a line connecting variable node $u_{k}$ and function node $c_{n}$. 
The generic form of CL iterated MUD can explained as follow. Let $\mathcal{L}_{n \downarrow}^{j}\left(x_{k}\right)$ and $\mathcal{L}_{n \uparrow}^{j}\left(x_{k}\right)$ be the messages ${ }^{1}$ sent along edge $e_{n, k}$, at $j$-th iteration, from variable node $u_{k}$ to function node $c_{n}$ and vice-versa, respectively. Assuming there is no a priori probabilities available, the initial messages $(j=0)$ are set to zeros: $\mathcal{L}_{n \downarrow}^{0}\left(x_{k}\right)=0, \forall k, \forall n$. The messages are updated using the following rules:

$$
\begin{aligned}
\mathcal{L}_{n \downarrow}^{j}\left(x_{k}\right) & =\sum_{m \in \xi_{k} \backslash n} \mathcal{L}_{m \uparrow}^{j-1}\left(x_{k}\right) \\
\mathcal{L}_{n \uparrow}^{j}\left(x_{k}\right) & =\mathcal{F}\left(x_{k} \mid y_{n}, \mathcal{L}_{n \downarrow}^{j}\left(x_{l}\right), \forall l \in \zeta_{k} \backslash k\right)
\end{aligned}
$$

It can be easily seen from (3) and (4), that all messages are restricted to consist of only the extrinsic information [7].

To approximate the optimum MAP (or Log-MAP as the messages are represented in their logarithmic-values) detector, as derived in [5], the function in (4) must calculate the local marginalization and is given by

$$
\mathcal{F}(\bullet)=\log \left(\sum_{\substack{[n] \in \mathbb{X}^{d_{c}} \\ x_{k}}} p^{j}\left(y_{n} \mid \mathbf{x}^{[n]}\right) \prod_{l \in \xi_{n} \backslash k} P_{n}^{j}\left(x_{l}\right)\right)
$$

where

$$
\begin{aligned}
p^{j}\left(y_{n} \mid \mathbf{x}^{[n]}\right) & \propto \exp \left(-\frac{1}{\left(2 \sigma^{2}\right)}\left\|y_{n}-\mathbf{h}_{[n]}^{T} \mathbf{x}^{[n]}\right\|^{2}\right) \\
P_{n}^{j}\left(x_{l}\right) & =\exp \left(\mathcal{L}_{n \downarrow}^{j}\left(x_{l}\right)\right) .
\end{aligned}
$$

Combining (6) and (7) into (5), the message update is given by

$$
\mathcal{L}_{n \uparrow}^{j}\left(x_{k}\right) \propto \max _{\substack{\mathbf{x}^{[n]} \in \mathbb{X}^{d_{c}} \\ x_{k}}}\left(\sum_{l \in \xi_{n} \backslash k} \mathcal{L}_{n \downarrow}^{j}\left(x_{l}\right)-\frac{1}{2 \sigma^{2}}\left\|y_{n}-\mathbf{h}_{[n]}^{T} \mathbf{x}^{[n]}\right\|^{2}\right)
$$

where $\max ^{\star}=\log \left(e^{a}+e^{b}\right)=\max (a, b)+\ln (1+\exp (-\mid a-$ $\left.\left.b\right|^{2}\right)$ ) is the numerically stable function derived by using Jacobian algorithm in [8]. By using local marginalization, this technique is termed CLi Log-MAP (LM) MUD. Furthermore, it is easy to see that, by utilizing local marginalization, the complexity of the receiver has been reduced so that it is exponential only to $d_{c}$ instead of to $K$, where $d_{c} \ll k$.

\section{B. G-GF CLi MUD}

The new Grouped-based technique operates in the chip-level and works by arranging the interfering users in a chip into two groups. Let $G$ and $\bar{G}$ be sets of users for group 1 and group 2, correspondingly, such that $G \cap \bar{G}=\emptyset$. Furthermore, $|G|$ and $|\bar{G}|$ denote the size of each group, respectively. The idea is to approximate the Log-Likelihood Ratio (LLR) of the interference coming from other group as a single symmetric Gaussian distributed, so that (2) can be re-written as

$$
\begin{aligned}
y_{n} & =\sum_{k \in G} h_{k, n} x_{k}+\sum_{l \in \bar{G}} h_{l, n} x_{l}+\nu_{n} \\
& =\sum_{k \in G} h_{k, n} x_{k}+\operatorname{mai}_{G, n}+\nu_{n}
\end{aligned}
$$

\footnotetext{
${ }^{1}$ Message is defined as a vector comprising the reliability values of the adjacent variable node and is represented by its computationally efficient and stable Logarithmic-value.
}

Let $\tilde{x}_{k, n}$ and $\operatorname{Var}\left\{x_{k, n}\right\}$ be the mean and the variance of the symbol $x_{k}$ when observing at chip $r_{n}$, respectively, and are given by

$$
\begin{aligned}
\tilde{x}_{k, n} & =\sum_{x_{k} \in \mathbb{X}} x_{k} P_{n}\left(x_{k}\right) \\
\operatorname{Var}\left(x_{k, n}\right) & =\sum_{x_{k} \in \mathbb{X}}\left\|x_{k}\right\|^{2} P_{n}\left(x_{k}\right)-\left\|\widetilde{x}_{k, n}\right\|^{2} .
\end{aligned}
$$

Then, employing (10) and (11), when observing the users from group $G$, the cumulative statistics of interference from another group $\bar{G}$ can written as

$$
\begin{aligned}
\mathrm{E}\left\{\operatorname{mai}_{G, n}\right\} & =\sum_{k \in \bar{G}} h_{k, n} \tilde{x}_{k, n} \\
\operatorname{Var}\left(\operatorname{mai}_{G, n}\right) & =\sum_{k \in \bar{G}}\left\|h_{k, n}\right\|^{2} \operatorname{Var}\left(x_{k, n}\right) .
\end{aligned}
$$

In line with the investigation given in [9] that states parallel processing is more advantageous than serial processing where user/group ordering is much less sensitive, the same treatment is made when evaluating the group $\bar{G}$.

Let $\sigma_{G, n}^{2}=\sigma_{n}^{2}+\operatorname{Var}\left(\operatorname{mai}_{G, n}\right)$ be the cumulative noise variance seen by users in group $G$. By using the above statistics, the MAP-based CLi SISO MUD techniques can be simplified. Denote $\mathbf{x}^{[G, n]}$ and $\mathbf{h}_{[G, n]}$ as the vectors comprising all users' symbols in group $\mathrm{G}$ and their corresponding effective signature vector, respectively. By using the Log-MAP algorithm as the local observation function, (8) can be modified according to the Gaussian approximation of the interferers and is given by

$$
\begin{aligned}
\mathcal{L}_{n \uparrow}^{j}\left(x_{k}\right) & \propto \max _{\substack{\mathbf{x}^{[n]} \in \mathbb{X}|G| \\
x_{k}}}\left(\sum_{l \in G \backslash k} \mathcal{L}_{n \downarrow}^{j}\left(x_{l}\right)-\right. \\
& \left.\frac{1}{2 \sigma_{G, n}^{2}}\left\|y_{n}-\mathbf{h}_{[G, n]}^{T} \mathbf{x}^{[G, n]}-\mathrm{E}\left\{\operatorname{mai}_{G, n}\right\}\right\|^{2}\right) .
\end{aligned}
$$

Assuming that the size of $G$ is bigger than that of $\bar{G}$, it is easy to see from (14) that the complexity of this new detection is exponential to $|G|<\xi_{n}$ instead of to $\xi_{n}$. Note that, by forcing the interference from other group as single symmetric Gaussian distribution when it is actually incorrect, some performance loss is expected when this new technique is employed.

Moreover, another key contribution to this G-GF CLi SISO MUD techniques is the utilization of dynamic random user grouping that dynamically randomize/shuffle the users from both groups in every iteration. In other words, the users grouping is done for every iteration and the exact same set of users are not allowed to form the same group during two consecutive iterations. By doing that, the intra-relation that may be developed between users in a group can be broken down. In a way, it provides the extrinsic information in every iteration to calculate the new message. And as stated in [10], the extrinsic information can help increasing the optimality of MPA technique in the sense that it provides more search space.

\section{Non-Linear Smoothing Process}

The CLi MUD techniques given in previous sub-sections may suffer from the oscillatory and, even, chaotic condition 


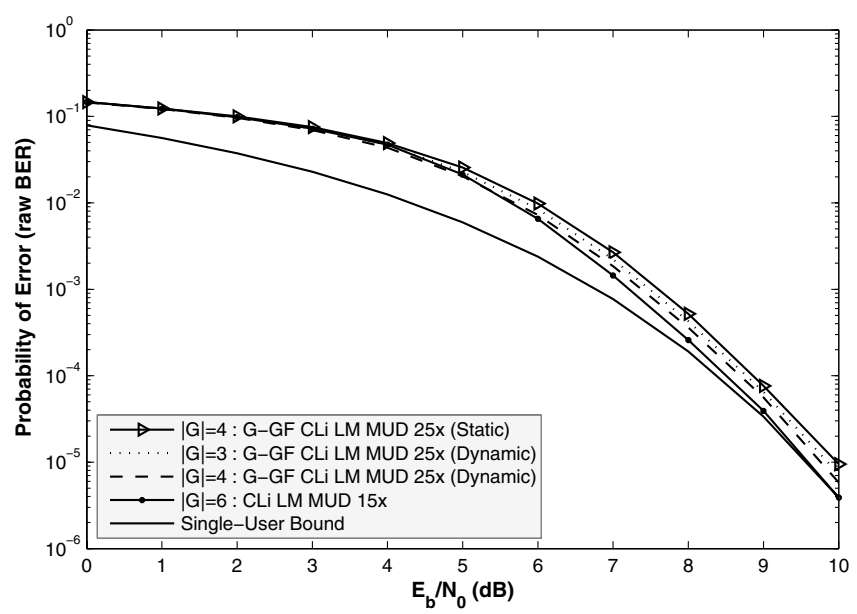

Fig. 1. Effect of various group size and different user grouping strategy for G-GF CLi LM MUD.

[11]. These phenomena emerge due to the failure of the iterative receivers to reach its fixed points, i.e. the nonconvergent case. In this paper, we propose a simple non-linear smoothing process to bound the future estimate such that it will not lie too far from the previous estimations and is given by

$$
\mathcal{L}_{n \uparrow}^{j}\left(x_{k}\right) \propto\left(\alpha \mathcal{L}_{n \uparrow}^{j}\left(x_{k}\right)+(1-\alpha) \mathcal{L}_{n \uparrow}^{j-1}\left(x_{k}\right)\right)
$$

where $\alpha$ is the smoothing coefficient. Note that the normalization of the message shall be done before as well as after the smoothing process. Furthermore, during our initial investigation, we have found out that for the same LDS scheme and the same CL iterative MUD technique, the smoothing coefficients are not too sensitive to the SNR.

\section{Simulation Results}

The regular LDS $60 \times 120$ is designed to serve up to $K=$ 120 users with $N=60$ chips with effective spreading $d_{v}=3$ and number of interfering users in a chip $d_{c}=6$. Before, we show the performance of the proposed G-GF CLi LM MUD, we review the necessity of incorporating non-linear smoothing process into chip observation function.

We present the superiority of the dynamic user grouping when used in G-GF CLi LM MUD in Figure 1. Since the non-linear smoothing process slows down the convergence, a greater number of iteration is needed for the G-GF CLi LM MUD with smaller group size. Specifically shown in Figure 1, for G-GF CLi LM MUD with $|G|<5$, the maximum number of iteration is limited to 25 , otherwise 15 . Furthermore, it is also shown that the performance loss when G-GF CLi LM MUD with $|G|=3$ is employed is approximately 0.3 $\mathrm{dB}$ compared when brute-force CLi LM MUD is utilized. Moreover, by increasing the group size, as suggested by our results, the performance should be improved. Interestingly, we also observed that by employing dynamic grouping, G-GF CLi LM MUD witg $|G|=3$ outperforms the G-GF CLi MUD employing static grouping with $|G|=4$.

Understandably, when $|G|$ is increased, the performance is also improved because the search space becomes larger. Also, due to the dynamic user grouping, all users basically will have the same treatment.

\section{CONCLUSION}

This letter studies the performance of a lower-complexity implementation of CLi LM MUD by using Gaussian-Forcing technique. Trading-off the performance and complexity, a new dynamic G-GF CLi LM MUD has been proposed and analyzed. Our simulation confirms that the performance loss incurred due to having smaller group size can be justified given the achieved complexity reduction.

\section{REFERENCES}

[1] D. Tse and S. Hanly, "Linear multiuser receivers: Effective interference, effective bandwidth and user capacity," IEEE Trans. Inform. Theory, vol. 45, no. 2, pp. 641-657, Mar. 1999.

[2] S. Verdú, Multiuser Detection. New York: Cambridge Univ. Press, 1998.

[3] L. Welch, "Lower bounds on the maximum cross-correlation of signals," IEEE Trans. Inform. Theory, vol. 20, pp. 397-399, 1974.

[4] M. Varanasi, C. Mullis, and A. Kapur, "On the limitation of linear MMSE detection," IEEE Trans. Inform. Theory, vol. 52, no. 9, pp. 42824286, Sept. 2006.

[5] R. Hoshyar, F. Wathan, and R. Tafazolli, "Novel low-density signature structure for synchronous DS-CDMA systems," in Proc. IEEE Globecom'06, San Francisco, CA, Nov. 2006, pp. 1-5.

[6] _ - "Novel low-density signature for synchronous CDMA systems over AWGN channel," accepted for publication by IEEE Trans. Signal Processing.

[7] F. Kschischang, B. Frey, and H. Loeliger, "Factor graphs and the sumproduct algorithm," IEEE Trans. Inform. Theory, vol. 47, no. 2, pp. 498-519, Feb. 2001.

[8] A. Viterbi, "An intuitive justification and a simplified implementation of the MAP decoder for convolutional codes," IEEE J. Select. Areas Commun., vol. 16, no. 2, pp. 260-264, Feb. 1998.

[9] F. Wathan, R. Hoshyar, and R. Tafazolli, "Iterated siso mud for synchronous uncoded cdma systems over awgn channel: Performance evaluation in overloaded condition," in Proc. Int. Symposium Commun. and Inform. Technologies (ISCIT) 2007, Sydney, Australia, Oct. 2007.

[10] T. Richardson and R. Urbanke, "The capacity of low-density paritycheck codes under message-passing decoding," IEEE Trans. Inform. Theory, vol. 47, no. 2, pp. 599-618, Feb. 2001.

[11] L. Kocarev et al., "Nonlinear dynamics of iterative decoding systems: analysis and applications," IEEE Trans. Inform. Theory, vol. 52, no. 4, pp. 1366-1384, Apr. 2006. 